\title{
The elementary obstruction and the Weil restriction
}

\author{
Tim Wouters
}

October 2, 2008

\begin{abstract}
In this text we investigate the good behaviour of the elementary obstruction, introduced by Colliot-Thélène and Sansuc [3]. This is an obstruction to the existence of a rational points on certain algebraic varieties. Assuming some conditions on the Picard group, we prove that the elementary obstruction behaves well under the Weil restriction of a variety.
\end{abstract}

\section{Introduction}

For a field $k$ and a variety $X$ over $k$ (i.e. a separated $k$-scheme of finite type), questions concerning $k$-rational points of $X$ have been studied since ages. Different aspects arise in this area of research. In this paper we will focus on a certain obstruction to the existence of a rational point, namely the elementary obstruction, introduced by Colliot-Thélène and Sansuc [3, Sec. 2.2].

Let $\bar{k}$ be a separable closure of $k$ and $\Gamma=\operatorname{Gal}(\bar{k} / k)$. If $X$ is a smooth, geometrically integral variety over $k$, the elementary obstruction $\mathrm{ob}(X)$ of $X$ is defined as the class of the exact sequence of left $\Gamma$-modules

$$
\mathrm{OB}(X):=1 \rightarrow \bar{k}^{\times} \rightarrow \bar{k}(X)^{\times} \rightarrow \bar{k}(X)^{\times} / \bar{k}^{\times} \rightarrow 1
$$

in $\operatorname{Ext}_{\Gamma}^{1}\left(\bar{k}(X)^{\times} / \bar{k}^{\times}, \bar{k}^{\times}\right)$. Note that we use the common notation $\bar{k}(X)$ for the function field of $\bar{X}=X \times_{k} \bar{k}$. Analogously, we will denote $\bar{k}[X]$ to be ring of regular functions on $\bar{X}$. If $X$ contains a $k$-rational point, then $\mathrm{ob}(X)=0$ [3, Prop. 2.2.2]. Furthermore, if $\bar{k}[X]^{\times}=\bar{k}^{\times}$, the class of

$$
E(X):=1 \rightarrow \bar{k}^{\times} \rightarrow \bar{k}(X)^{\times} \rightarrow \operatorname{Div}(\bar{X}) \rightarrow \operatorname{Pic}(\bar{X}) \rightarrow 1
$$

K.U.Leuven - Departement Wiskunde, Celestijnenlaan 200B bus 2400, B-3001 Heverlee, Belgium, tim@wouters.in

2000 Mathematics Subject Classification: 14G05, 11G99

Keywords: Elementary obstruction - Weil restriction - Rational points

Dedicated to the memory of Joost van Hamel, for all trouble he took to support this article with discussions, comments and suggestions at all times, also in moments when it is not obvious that one keeps on focusing on mathematics. 
in $\operatorname{Ext}_{\Gamma}^{2}\left(\operatorname{Pic}(\bar{X}), \bar{k}^{\times}\right)$is denoted by $e(X)$. Colliot-Thélène and Sansuc showed that the morphism

$$
\delta: \operatorname{Ext}_{\Gamma}^{1}\left(\bar{k}(X)^{\times} / \bar{k}^{\times}, \bar{k}^{\times}\right) \rightarrow \operatorname{Ext}_{\Gamma}^{2}\left(\operatorname{Pic}(\bar{X}), \bar{k}^{\times}\right),
$$

which arises in the long exact sequence induced by

$$
1 \rightarrow \bar{k}(X)^{\times} / \bar{k}^{\times} \rightarrow \operatorname{Div}(\bar{X}) \rightarrow \operatorname{Pic}(\bar{X}) \rightarrow 1,
$$

is injective and that $\delta(\mathrm{ob}(X))=e(X)$ [3. Prop. 2.2.4]. This is a consequence of Shapiro's Lemma and Hilbert 90. Therefore it is also justified to say $e(X)$ is the elementary obstruction of $X$. In this paper we will mainly use this definition for the elementary obstruction.

Several authors have been wondering whether the elementary obstruction behaves well under classical geometric constructions. A first observation is that the elementary obstruction is a birational invariant, since birationally equivalent varieties have isomorphic function fields. Wittenberg proved being zero behaves well under rational maps [9, Lem. 3.1.2]. Borovoi, Colliot-Thélène and Skorobogatov wondered whether being zero behaves well under base extension (i.e. whether $\mathrm{ob}(X)=0 \mathrm{im}$ plies ob $\left(X \times_{k} K\right)=0$ for $K \supset k$ and $X$ a smooth, geometrically integral variety over $k)$ [1, Sec. 2]. They gave several (partial) positive answers to this question. Wittenberg gave a positive answer to this question for arbitrary (smooth, proper, geometrically integral) $X$ when $K$ is a $p$-adic or real closed field [9, Cor. 3.2.3] or when $k$ is a number field and the Tate-Shafarevich group of the Picard variety of $X$ is finite [9, Cor. 3.3.2]. But recently he gave a negative answer to this question by producing a counterexample over $\mathbb{C}((t))$ (not published yet).

In this paper we focus on the question whether being zero behaves well under the Weil restriction of varieties. To describe the problem more explicitely, we first recall the definition of the Weil restriction.

Definition 1. Let $k$ be a field and $k^{\prime}$ a finite field extension of $k$. Let $X$ be a variety defined over $k^{\prime}$. We say a variety $\mathcal{R}_{k^{\prime} / k} X$ over $k$ is the Weil restriction of $X$ if there is a $k^{\prime}$-morphism $\varphi: \mathcal{R}_{k^{\prime} / k} X \times_{k} k^{\prime} \rightarrow X$ such that for any $k$-variety $Y$ and $k^{\prime}$-morphism $f: Y \times_{k} k^{\prime} \rightarrow X$, a unique $k$-morphism $g: Y \rightarrow \mathcal{R}_{k^{\prime} / k} X$ exists such that $\varphi \circ g^{\prime}=f$, where $g^{\prime}: Y \times_{k} k^{\prime} \rightarrow \mathcal{R}_{k^{\prime} / k} X \times_{k} k^{\prime}$ is the $k^{\prime}$-morphism induced by $g$. If the Weil restriction exists, it is unique up to $k$-isomorphism.

There is a well known proposition that guarantees the existence of the Weil restriction under the assumption of some conditions.

Proposition 2. Let $k$ be a field, $\bar{k}$ a separable closure and $k^{\prime}$ a finite subextension of $k$ in $\bar{k}$. Denote $\Gamma=\operatorname{Gal}(\bar{k} / k), H=\operatorname{Gal}\left(\bar{k} / k^{\prime}\right)$ and let $X$ be a quasiprojective variety over $k^{\prime}$. The Weil restriction $\mathcal{R}_{k^{\prime} / k} X$ of $X$ exists, and

$$
\mathcal{R}_{k^{\prime} / k} X \times_{k} \bar{k}=\prod_{[\sigma] \in H \backslash \Gamma} \sigma X
$$

where $\sigma X$ is the $\bar{k}$-variety obtained by base extension of $X \times_{k} \bar{k}$ by $\sigma: \bar{k} \rightarrow \bar{k}$ and $H \backslash \Gamma$ are the right cosets of $H$ in $\Gamma$. The $k^{\prime}$-morphism $\varphi: \mathcal{R}_{k^{\prime} / k} X \times_{k} k^{\prime} \rightarrow X$ is obtained by descent theory from its base extension $\bar{\varphi}: \overline{\mathcal{R}_{k^{\prime} / k} X} \rightarrow \bar{X}$, which is the projection onto the factor $(i d) X$.

For the proof we refer to [7, Prop. 16.26]. Remark that if $[\sigma]=[\tau] \in \Gamma / H$, the universal property of fibre products garantuees $\sigma X$ and $\tau X$ to be isomorphic as $\bar{k}$-varieties. 
The universal property of the Weil restriction implies also a 1-1 correspondence between $\mathcal{R}_{k^{\prime} / k} X(k)$ and $X\left(k^{\prime}\right)$, since rational points are equivalent with sections of the structure morphism. As there is a natural connection between rational points of a variety and its Weil restriction, it is natural to ask the following question.

Question 3. Let $k$ be a field and $k^{\prime}$ a finite field extension. Suppose $X$ is a smooth, geometrically integral variety over $k^{\prime}$ such that the Weil restriction $\mathcal{R}_{k^{\prime} / k} X$ exists. Does $e(X)=0$ implies $e\left(\mathcal{R}_{k^{\prime} / k} X\right)=0$ and vice versa?

We will answer this question partially positively (Proposition 10 and Theorem 13 , but first we will give a result on the elementary obstruction of a product variety (Theorem 4), as Proposition 2 tells us that the Weil restriction is closely related to product varieties.

\section{Product varieties}

In this section let $k$ be a field, $\bar{k}$ a separable closure and $\Gamma=\operatorname{Gal}(\bar{k} / k)$. Let $X$ and $Y$ be two smooth geometrically integral varieties over $k$, then the following theorem is a merely homological result.

Theorem 4. The multiplication $\Gamma$-morphism $\pi: \bar{k}(X)^{\times} / \bar{k}^{\times} \oplus \bar{k}(Y)^{\times} / \bar{k}^{\times} \rightarrow \bar{k}\left(X \times_{k}\right.$ $Y)^{\times} / \bar{k}^{\times}$induces a morphism by pullback

$$
\begin{aligned}
& \pi^{* \prime}: \operatorname{Ext}_{\Gamma}^{1}\left(\bar{k}\left(X \times_{k} Y\right)^{\times} / \bar{k}^{\times}, \bar{k}^{\times}\right) \rightarrow \\
& \quad \operatorname{Ext}_{\Gamma}^{1}\left(\bar{k}(X)^{\times} / \bar{k}^{\times}, \bar{k}^{\times}\right) \oplus \operatorname{Ext}_{\Gamma}^{1}\left(\bar{k}(Y)^{\times} / \bar{k}^{\times}, \bar{k}^{\times}\right)
\end{aligned}
$$

such that $\pi^{* \prime}\left(o b\left(X \times_{k} Y\right)\right)=(o b(X), o b(Y))$. If $\bar{k}[X]^{\times}=\bar{k}^{\times}=\bar{k}[Y]^{\times}$, then the $\Gamma$-morphism $\psi: \operatorname{Pic}(\bar{X}) \oplus \operatorname{Pic}(\bar{Y}) \rightarrow \operatorname{Pic}\left(\bar{X} \times_{\bar{k}} \bar{Y}\right)$ defined by pullback of line bundles, induces a morphism

$$
\psi^{* \prime}: \operatorname{Ext}_{\Gamma}^{2}\left(\operatorname{Pic}\left(\bar{X} \times_{\bar{k}} \bar{Y}\right), \bar{k}^{\times}\right) \rightarrow \operatorname{Ext}_{\Gamma}^{2}\left(\operatorname{Pic}(\bar{X}), \bar{k}^{\times}\right) \oplus \operatorname{Ext}_{\Gamma}^{2}\left(\operatorname{Pic}(\bar{Y}), \bar{k}^{\times}\right)
$$

such that $\psi^{* \prime}\left(e\left(X \times_{k} Y\right)\right)=(e(X), e(Y))$. Even more $\pi^{* \prime}$ and $\psi^{* \prime}$ commute with the natural inclusions in the following commutative diagram:

$$
\begin{aligned}
& \left.\right|_{\delta} ^{\operatorname{Ext}_{\Gamma}^{1}\left(\bar{k}\left(X \times_{k} Y\right)^{\times} / \bar{k}^{\times}, \bar{k}^{\times}\right) \pi_{\pi^{* \prime}}} \operatorname{Ext}_{\Gamma}^{1}\left(\bar{k}(Y)^{\times} / \bar{k}^{\times}, \bar{k}^{\times}\right) \oplus \operatorname{Ext}_{\Gamma}^{1}\left(\bar{k}(Y)^{\times} / \bar{k}^{\times}, \bar{k}^{\times}\right)
\end{aligned}
$$

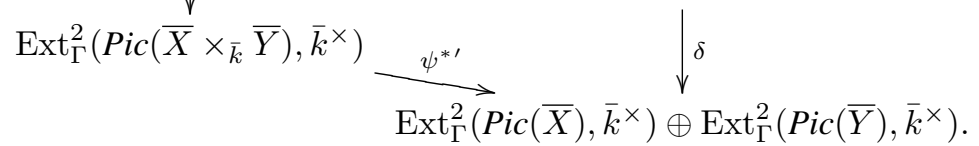

If $\pi$ or $\psi$ is an isomorphism, then $e\left(X \times{ }_{k} Y\right)=0\left(\right.$ resp. $\left.o b\left(X \times_{k} Y\right)=0\right)$ if and only if $e(X)=0$ and $e(Y)=0($ resp. $o b(X)=0$ and $o b(Y)=0)$.

Remark 5. Note that if $X$ and $Y$ are smooth geometrically integral varieties satisfying $\bar{k}[X]^{\times}=\bar{k}^{\times}=\bar{k}[Y]^{\times}$, then $X \times_{k} Y$ is also smooth geometrically integral, and by a result of Rosenlicht [8] Thm. 2] it satisfies $\bar{k}\left[X \times_{k} Y\right]^{\times}=\bar{k}^{\times}$. So speaking about $e\left(X \times_{k} Y\right)$ in the second case does make sense. 
Proof. If we denote the canonical isomorphism

$$
\begin{aligned}
\operatorname{Ext}_{\Gamma}^{1}\left(\bar{k}(X)^{\times} / \bar{k}^{\times} \oplus \bar{k}(Y)^{\times} / \bar{k}^{\times}, \bar{k}^{\times}\right) \cong \\
\operatorname{Ext}_{\Gamma}^{1}\left(\bar{k}(X)^{\times} / \bar{k}^{\times}, \bar{k}^{\times}\right) \oplus \operatorname{Ext}_{\Gamma}^{1}\left(\bar{k}(Y)^{\times} / \bar{k}^{\times}, \bar{k}^{\times}\right)
\end{aligned}
$$

by $\varphi$, then $\pi^{* \prime}:=\varphi \circ \pi^{*}$ is the required morphism, where

$$
\pi^{*}: \operatorname{Ext}_{\Gamma}^{1}\left(\bar{k}\left(X \times_{k} Y\right)^{\times} / \bar{k}^{\times}, \bar{k}^{\times}\right) \rightarrow \operatorname{Ext}_{\Gamma}^{1}\left(\bar{k}(X)^{\times} / \bar{k}^{\times} \oplus \bar{k}(Y)^{\times} / \bar{k}^{\times}, \bar{k}^{\times}\right)
$$

is the pullback of 1-extensions by $\pi$. We now prove the assertion on the elementary obstruction.

We surely have a morphism of short exact sequences which consists of product morphisms:

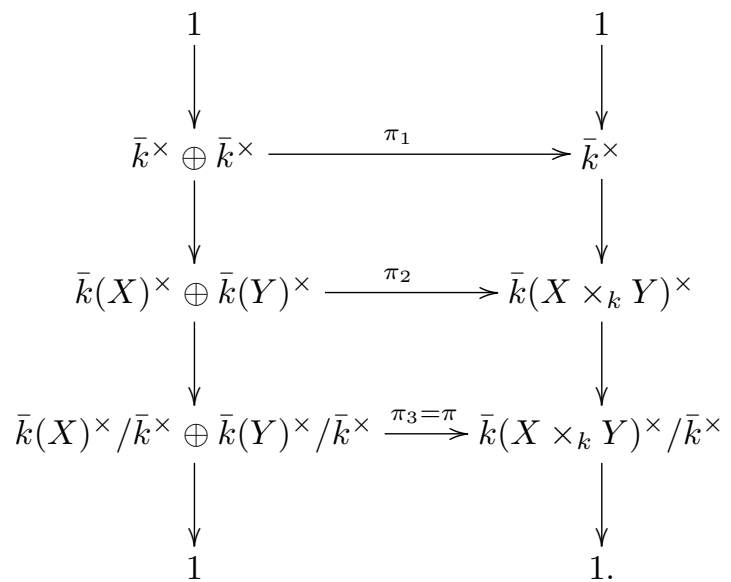

Denote the left short exact sequence by $E(X) \oplus E(Y)$. By the notation introduced in the introduction, the right short exact sequence is denoted by $E\left(X \times_{k} Y\right)$. By the general theory of Yoneda extensions [6, Ch. III], we get

$$
\varphi^{-1}(e(X), e(Y))=\left[\pi_{1}(E(X) \oplus E(Y))\right]=\left[E\left(X \times_{k} Y\right) \pi_{3}\right]=\pi^{*}\left(e\left(X \times_{k} Y\right)\right),
$$

where $\pi_{1}(E(X) \oplus E(Y))$ denotes the pushforward of the Yoneda extension $E(X) \oplus$ $E(Y)$ by $\pi_{1}$ and $E\left(X \times_{k} Y\right) \pi_{3}$ denotes the pullback of the Yoneda extension $E\left(X \times_{k}\right.$ $Y)$ by $\pi_{3}$. This proves the first part.

The second part is proved analogously, using $\Gamma$-morphisms $\pi_{4}: \operatorname{Div}(\bar{X}) \oplus \operatorname{Div}(\bar{Y}) \rightarrow$ $\operatorname{Div}\left(\bar{X} \times_{\bar{k}} \bar{Y}\right)$ and $\psi: \operatorname{Pic}(\bar{X}) \oplus \operatorname{Pic}(\bar{Y}) \rightarrow \operatorname{Pic}\left(\bar{X} \times{ }_{\bar{k}} \bar{Y}\right)$. The commutativity assertion follows from the following morphism of short exact sequences 


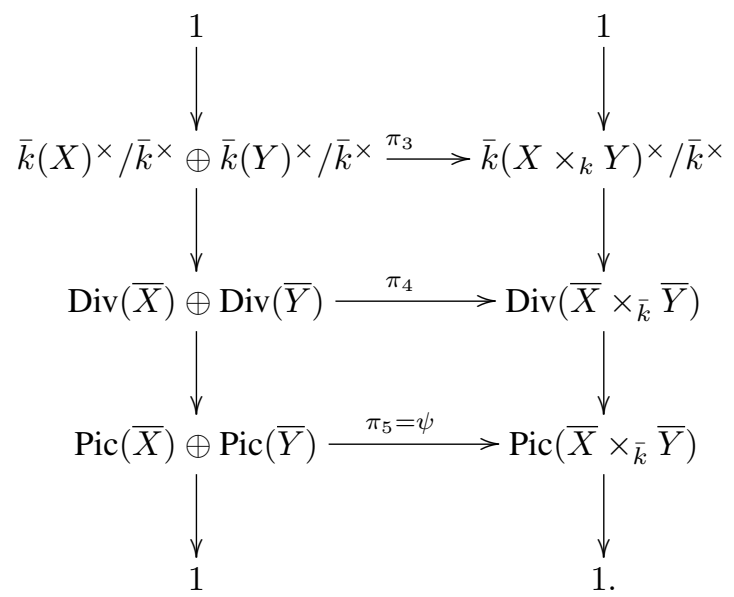

which induces a morphism of long exact sequences, by Shapiro's lemma and Hilbert 90 containing the required diagram.

So we see that in any case $e(X)=0$ and $e(Y)=0$ (resp. $\mathrm{ob}(X)=0$ and $\mathrm{ob}(Y)=0)$ if $e(X \times Y)=0$ (resp. $\mathrm{ob}(X \times Y)=0$ ). If $\psi$ (resp. $\pi$ ) is an isomorphism, $\psi^{* \prime}$ (resp. $\left.\pi^{* \prime}\right)$ will be so too, so in one of these cases the inverse implication holds as well (recall that $e(-)=0$ if and only if $\mathrm{ob}(-)=0$ ).

Remark 6. A known result says that if $\bar{X}$ and $\bar{Y}$ are varieties over separable closed field $\bar{k}$, then as groups the morphism $\psi: \operatorname{Pic}(\bar{X}) \oplus \operatorname{Pic}(\bar{Y}) \rightarrow \operatorname{Pic}\left(\bar{X} \times_{\bar{k}} \bar{Y}\right)$, defined by pull-backs, has a section. This section restricts a line bundle on $X \times_{k} Y$ to $x_{0} \times Y$ and $X \times y_{0}$ where $x_{0}$ and $y_{0}$ are base points on $X$ and $Y$. So as groups $\operatorname{Pic}(\bar{X}) \oplus \operatorname{Pic}(\bar{Y})$ is a direct summand of Pic $\left(\bar{X} \times_{\bar{k}} \bar{Y}\right)$. This looks interesting to get more information on the structure of $\operatorname{Ext}_{\Gamma}^{2}\left(\operatorname{Pic}\left(\bar{X} \times_{\bar{k}} \bar{Y}\right), \bar{k}^{\times}\right)$. In our case however, $X$ and $Y$ are defined over a not necessarily separably closed field $k$ and $\psi: \operatorname{Pic}(\bar{X}) \oplus \operatorname{Pic}(\bar{Y}) \rightarrow \operatorname{Pic}\left(\bar{X} \times_{\bar{k}} \bar{Y}\right)$ is $a \Gamma$-morphism, but the section is not necessarily a $\Gamma$-morphism since the base points do not have to behave well (if we do not know anything about the existence of k-rational points on $X$ and $Y$ ). So we can not use this result to extend the previous theorem in a direct way. However, we do retrieve $\psi$ is injective.

Off course $\psi: \operatorname{Pic}(\bar{X}) \oplus \operatorname{Pic}(\bar{Y}) \rightarrow \operatorname{Pic}\left(\bar{X} \times_{\bar{k}} \bar{Y}\right)$ does not need to be an isomorphism, the product of an elliptic curve with itself delivering a counterexample [5, Ch. IV, Ex. 4.10]. We can however give sufficient conditions for $\psi$ to be an isomorphism. This will involve the notion of the relative Picard functor and the Picard variety. If $X$ is a smooth, geometrically integral, projective variety over a field $k$, we denote the relative Picard functor by $\mathcal{P} \mathrm{ic}_{X / k}$, which is representable by a group variety $\operatorname{Pic}(X)$, the Picard variety. Denote by $\mathbf{P i c}^{0}(X)$ the zerocomponent of $\mathbf{P i c}(X)$. (See [2, Ch. 8] for more information.)

Proposition 7. If $X$ is projective and $\operatorname{Pic}^{0}(\bar{X})=0$, then $\psi: \operatorname{Pic}(\bar{X}) \oplus \operatorname{Pic}(\bar{Y}) \rightarrow$ $\operatorname{Pic}\left(\bar{X} \times_{\bar{k}} \bar{Y}\right)$ is a $\Gamma$-isomorphism.

Proof. By Remark 6 we know that $\psi$ is injective, so it is sufficient to prove coker $\psi=$ 0 . By definition

$$
\operatorname{Pic}_{\bar{X} / \bar{k}}(\bar{Y})=\operatorname{Pic}\left(\bar{X} \times_{\bar{k}} \bar{Y}\right) / \operatorname{Pic}(\bar{Y}) \cong \operatorname{Hom}_{\bar{k}}(\bar{Y}, \operatorname{Pic}(\bar{X})) .
$$


Any $f \in \operatorname{Hom}_{\bar{k}}(\bar{Y}, \operatorname{Pic}(\bar{X}))$ has a connected image, but since $\mathbf{P i c}^{0}(\bar{X})=0$, the connected components of $\operatorname{Pic}(\bar{X})$ are its points. So $\operatorname{Hom}_{\bar{k}}(\bar{Y}, \operatorname{Pic}(\bar{X}))$ consists of the constant maps onto a point of $\operatorname{Pic}(\bar{X})$. This does not depend on $Y$, so

$$
\operatorname{Hom}_{\bar{k}}(\bar{Y}, \operatorname{Pic}(\bar{X})) \cong \operatorname{Hom}_{\bar{k}}(\bar{k}, \operatorname{Pic}(\bar{X})) \cong \operatorname{Pic}(\bar{X}) .
$$

Because these isomorphisms are induced by the representability of the Picard functor,

$$
\operatorname{coker} \psi=\frac{\operatorname{Pic}\left(\bar{X} \times_{\bar{k}} \bar{Y}\right) / \operatorname{Pic}(\bar{Y})}{\operatorname{Pic}(\bar{X})}=\frac{\operatorname{Pic}(\bar{X})}{\operatorname{Pic}(\bar{X})}=0 \text {. }
$$

Proposition 8. If $X$ is quasiprojective, char $(k)=0$ and Pic $(\bar{X})$ is finitely generated, then $\operatorname{Pic}(\bar{X}) \oplus \operatorname{Pic}(\bar{Y}) \cong \operatorname{Pic}\left(\bar{X} \times_{\bar{k}} \bar{Y}\right)$.

Proof. Say $X \subset X_{1}$ for a projective variety $X_{1}$. Since $\operatorname{char}(k)=0$, there exists a (smooth, projective) Hironaka desingularisation $X^{\prime}$ of $X_{1}$. As $X$ is smooth, $X$ is isomorphic to an open of $X^{\prime}$. So without loss of generality we assume $X$ is an open part of $X^{\prime}$. The exact sequence

$$
\operatorname{Div}_{\overline{X^{\prime}} \backslash \bar{X}}\left(\overline{X^{\prime}}\right) \rightarrow \operatorname{Pic}\left(\overline{X^{\prime}}\right) \rightarrow \operatorname{Pic}(\bar{X}) \rightarrow 0
$$

induces $\operatorname{Pic}\left(\overline{X^{\prime}}\right)$ to be finitely generated, as $\operatorname{Pic}(\bar{X})$ and $\operatorname{Div}_{\overline{X^{\prime}}} \backslash \bar{X}(\bar{X})$ are finitely generated. (Div $\overline{X^{\prime}} \backslash \bar{X}(\bar{X})$ are the divisors on $\overline{X^{\prime}}$ with support outside $\bar{X}$.)

It suffices to prove $\operatorname{Pic}\left(\overline{X^{\prime}} \times_{\bar{k}} \bar{Y}\right) \cong \operatorname{Pic}\left(\overline{X^{\prime}}\right) \oplus \operatorname{Pic}(\bar{Y})$ as this will induce $\operatorname{Pic}\left(\bar{X} \times_{\bar{k}}\right.$ $\bar{Y}) \cong \operatorname{Pic}(\bar{X}) \oplus \operatorname{Pic}(\bar{Y})$. Indeed, there is a commutative diagram

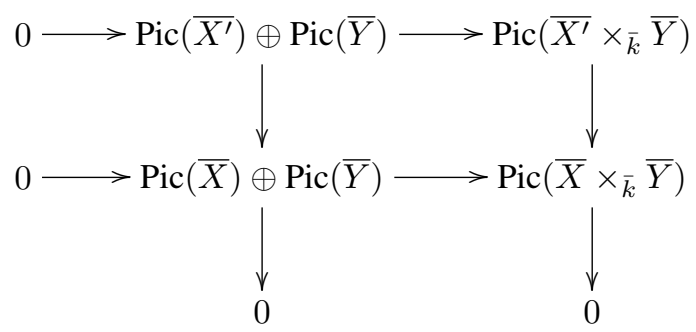

where the vertical arrows are the surjective restriction morphisms. If the injection of the first row turns out to be an isomorphism, then the injection of the bottom row should also be surjective, hence an isomorphism.

Because $\operatorname{Pic}\left(\overline{X^{\prime}}\right)$ is finitely generated, we have $\mathbf{P i c}^{0}\left(\overline{X^{\prime}}\right)=0$. Indeed, if $\mathbf{P i c}^{0}\left(\overline{X^{\prime}}\right) \neq$ 0 , then $\mathbf{P i c}^{0}\left(\overline{X^{\prime}}\right)$ is an abelian variety of dimension $m>0$ whose group of $\bar{k}$-points is finitely generated as $\operatorname{Pic}\left(\overline{X^{\prime}}\right)=\operatorname{Hom}_{\bar{k}}\left(\bar{k}, \operatorname{Pic}\left(\overline{X^{\prime}}\right)\right)$ is finitely generated. On the other hand the group of $\bar{k}$-points of an abelian variety is divisible [4, Thm. 2]. But a divisible, non-trivial, finitely generated group does not exist. In this way we get a contradiction and so the proposition follows by Proposition 7 .

Consequently we obtain the following result.

Corollary 9. Let $X$ and $Y$ be a smooth, geometrically integral varieties over a field $k$ with $\bar{k}[X]^{\times}=\bar{k}^{\times}=\bar{k}[Y]^{\times}$. Let $\bar{k}$ be a separable closure of $k$ and $\Gamma=\operatorname{Gal}(\bar{k} / k)$. If one of the following conditions holds 
(i) $X$ is projective and Pic $^{0}(\bar{X})=0$, or

(ii) $X$ is quasiprojective, char $(k)=0$ and Pic $(\bar{X})$ is finitely generated,

then

$$
\psi^{* \prime}: \operatorname{Ext}_{\Gamma}^{2}\left(\operatorname{Pic}\left(\bar{X} \times_{\bar{k}} \bar{Y}\right), \bar{k}^{\times}\right) \rightarrow \operatorname{Ext}_{\Gamma}^{2}\left(\operatorname{Pic}(\bar{X}), \bar{k}^{\times}\right) \oplus \operatorname{Ext}_{\Gamma}^{2}\left(\operatorname{Pic}(\bar{Y}), \bar{k}^{\times}\right)
$$

is an isomorphism such that $\psi^{*^{\prime}}\left(e\left(X \times_{k} Y\right)\right)=(e(X), e(Y))$.

So if one of the conditions is true, $e\left(X \times_{k} Y\right)=0$ if and only if $e(X)=0$ and $e(Y)=0$.

\section{Weil restriction}

Knowing more on the case of product varieties, we proceed to the Weil restriction. Throughout this section we will assume $k$ is a field, $\bar{k}$ is a separable closure of $k$ and $k^{\prime}$ is a finite subextension of $k$ in $\bar{k}$. Denote $\Gamma=\operatorname{Gal}(\bar{k} / k), H=\operatorname{Gal}\left(\bar{k} / k^{\prime}\right)$, and let $X$ be a smooth, geometrically integral, quasiprojective variety over $k^{\prime}$. In this case the Weil restriction of $X$ exists by Proposition 2 and we abbreviate it as $\mathcal{R}$.

Proposition 10. The natural $H$-morphism $\bar{k}(X)^{\times} \rightarrow \bar{k}(\mathcal{R})^{\times}$induces a pullback of 1-extensions

$$
\Pi^{*}: \operatorname{Ext}_{\Gamma}^{1}\left(\bar{k}(\mathcal{R})^{\times} / \bar{k}^{\times}, \bar{k}^{\times}\right) \rightarrow \operatorname{Ext}_{H}^{1}\left(\bar{k}(X)^{\times} / \bar{k}^{\times}, \bar{k}^{\times}\right),
$$

with $\Pi^{*}(o b(\mathcal{R}))=o b(X)$. If furthermore $\bar{k}[X]^{\times}=\bar{k}^{\times}$, then the natural H-morphism $\operatorname{Pic}(\bar{X}) \rightarrow \operatorname{Pic}(\overline{\mathcal{R}})$ induces a pullback of 2-extensions

$$
\Phi^{*}: \operatorname{Ext}_{\Gamma}^{2}\left(\operatorname{Pic}(\overline{\mathcal{R}}), \bar{k}^{\times}\right) \rightarrow \operatorname{Ext}_{H}^{2}\left(\operatorname{Pic}(\bar{X}), \bar{k}^{\times}\right),
$$

with $\Phi^{*}(e(\mathcal{R}))=e(X)$. As in Theorem 4 these morphisms commute with the natural inclusions sending ob(-) to $e(-)$.

Remark 11. The natural $H$-morphisms mentioned in the proposition are induced by Theorem 2 This proposition gives a $k^{\prime}$-morphism $\varphi: \mathcal{R} \times_{k} k^{\prime} \rightarrow X$ retrieved by descent from the $\bar{k}$-projection $\bar{\varphi}: \overline{\mathcal{R}} \rightarrow \bar{X}$. This morphism $\bar{\varphi}$ gives by pullback of principle divisors and line bundles the required $H$-morphisms.

Remark 12. As in Remark 5 it is true that $\bar{k}[\mathcal{R}]^{\times}=\bar{k}^{\times}$provided $\bar{k}[X]^{\times}=\bar{k}^{\times}$. So it makes sense to speak about $e(\mathcal{R})$ if at first glance we only require $\bar{k}[X]^{\times}=\bar{k}^{\times}$.

Proof. We give the proof of the assertion on 2-extensions. The assertion on 1-extensions follows in the same way, and the commutative part will follow as in Theorem 4

Denote the $H$-morphism $\operatorname{Pic}(\bar{X}) \rightarrow \operatorname{Pic}(\overline{\mathcal{R}})$ by $\varphi^{\prime}$. This induces a pullback

$$
\varphi^{\prime *}: \operatorname{Ext}_{H}^{2}\left(\operatorname{Pic}(\overline{\mathcal{R}}), \bar{k}^{\times}\right) \rightarrow \operatorname{Ext}_{H}^{2}\left(\operatorname{Pic}(\bar{X}), \bar{k}^{\times}\right) .
$$

If we use the forgetful map

$$
\pi: \operatorname{Ext}_{\Gamma}^{2}\left(\operatorname{Pic}(\overline{\mathcal{R}}), \bar{k}^{\times}\right) \rightarrow \operatorname{Ext}_{H}^{2}\left(\operatorname{Pic}(\overline{\mathcal{R}}), \bar{k}^{\times}\right),
$$

we get the required morphism $\Phi^{*}=\varphi^{*} \circ \pi$. 
To prove $\Phi^{*}(e(\mathcal{R}))=e(X)$, we use the following morphism of $H$-extensions

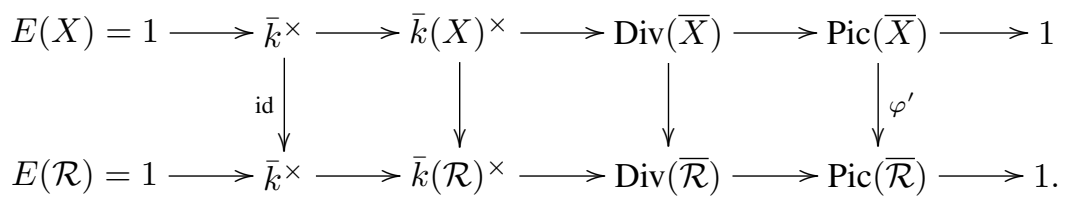

As it is clear that the $H$-equivalence class of $E(\mathcal{R})$ equals $\pi([e(\mathcal{R})])$, we get from elementary homological reasons

$$
\Phi^{*}(e(\mathcal{R}))=\varphi^{\prime *}(\pi([e(\mathcal{R})]))=[E(X)]=e(X) .
$$

So $e(\mathcal{R})=0$ implies $e(X)=0$. We proceed figuring out when the converse is true. This will hold in the very same situation as the converse holds for product varieties. To prove this, we will use the notion of induced group module, with some corresponding notation. Let $G$ be a profinite group, $H$ a subgroup of $G$ and $A$ a left $H$-module, then the induced $G$-module is $\operatorname{Ind}_{H}^{G}(A):=\mathbb{Z}[G] \otimes_{\mathbb{Z}[H]} A$, where $\mathbb{Z}[G]$ is considered as a right $\mathbb{Z}[H]$-module. This is a left $G$-module, the $G$-action is defined by $\gamma^{\prime}(\gamma \otimes a)=\gamma^{\prime} \gamma \otimes a$ for any $a \in A$ and $\gamma, \gamma^{\prime} \in G$. If $A$ and $B$ are left $H$-modules and $f: A \rightarrow B$ is an $H$-morphism, then we get an induced $G$-morphism

$$
\operatorname{Ind}_{H}^{G}(f): \operatorname{Ind}_{H}^{G}(A) \rightarrow \operatorname{Ind}_{H}^{G}(B) \quad \text { defined by } \quad \gamma \otimes a \mapsto \gamma \otimes f(a),
$$

for any $a \in A$ and $\gamma \in G$. If $B$ is also a left $G$-module, we write $\operatorname{Ind}_{H}^{G}(f)^{\prime}$ for the $G$-morphism $\pi \circ \operatorname{Ind}_{H}^{G}(f)$ with

$$
\pi: \operatorname{Ind}_{H}^{G}(B) \rightarrow B \quad \text { defined by } \quad \gamma \otimes b \mapsto{ }^{\gamma} b .
$$

If $E$ is an exact sequence

$$
A_{1} \stackrel{f_{1}}{\longrightarrow} A_{2} \stackrel{f_{2}}{\longrightarrow} A_{3}
$$

then we get an induced exact sequence $\operatorname{Ind}_{H}^{G}(E)$

$$
\operatorname{Ind}_{H}^{G}\left(A_{1}\right) \stackrel{\tilde{f}_{1}}{\longrightarrow} \operatorname{Ind}_{H}^{G}\left(A_{2}\right) \stackrel{\tilde{f}_{2}}{\longrightarrow} \operatorname{Ind}_{H}^{G}\left(A_{3}\right),
$$

where we have denoted $\tilde{f}_{i}:=\operatorname{Ind}_{H}^{G}\left(f_{i}\right)$ for sake of simplicity.

Theorem 13. If $\bar{k}[X]^{\times}=\bar{k}^{\times}$and if one of the two following conditions is true

(i) $X$ is projective and $\boldsymbol{P i c}^{0}(\bar{X})=0$, or

(ii) $X$ is quasiprojective, char $(k)=0$ and Pic $(\bar{X})$ is finitely generated,

then $\Phi^{*}$ of Proposition 10 is an isomorphism.

Proof. We will prove this result by giving another description of $\Phi^{*}$.

If $\varphi^{\prime}$ is the $H$-morphism $\operatorname{Pic}(\bar{X}) \rightarrow \operatorname{Pic}(\overline{\mathcal{R}})$ as defined in the proof of Proposition 10. the induced $\Gamma$-morphism $\operatorname{Ind}_{H}^{\Gamma}\left(\varphi^{\prime}\right)^{\prime}: \operatorname{Ind}_{H}^{\Gamma}(\operatorname{Pic}(\bar{X})) \rightarrow \operatorname{Pic}(\overline{\mathcal{R}})$ gives a pullback of 2-extensions,

$$
\operatorname{Ind}_{H}^{\Gamma}\left(\varphi^{\prime}\right)^{\prime *}: \operatorname{Ext}_{\Gamma}^{2}\left(\operatorname{Pic}(\overline{\mathcal{R}}), \bar{k}^{\times}\right) \rightarrow \operatorname{Ext}_{\Gamma}^{2}\left(\operatorname{Ind}_{H}^{\Gamma} \operatorname{Pic}(\bar{X}), \bar{k}^{\times}\right) .
$$


Furthermore say $\pi^{\prime}$ is the forgetful map

$$
\left.\left.\pi^{\prime}: \operatorname{Ext}_{\Gamma}^{2}\left(\operatorname{Ind}_{H}^{\Gamma}(\operatorname{Pic}(\bar{X})), \bar{k}^{\times}\right)\right) \rightarrow \operatorname{Ext}_{H}^{2}\left(\operatorname{Ind}_{H}^{\Gamma}(\operatorname{Pic}(\bar{X})), \bar{k}^{\times}\right)\right)
$$

and

$$
\left.\left.i^{*}: \operatorname{Ext}_{H}^{2}\left(\operatorname{Ind}_{H}^{\Gamma}(\operatorname{Pic}(\bar{X})), \bar{k}^{\times}\right)\right) \rightarrow \operatorname{Ext}_{H}^{2}\left(\operatorname{Pic}(\bar{X}), \bar{k}^{\times}\right)\right)
$$

the pullback by $i: \operatorname{Pic}(\bar{X}) \rightarrow \operatorname{Ind}_{H}^{\Gamma}(\operatorname{Pic}(\bar{X})): \mathcal{L} \mapsto$ id $\otimes \mathcal{L}$. So we end up in the following situation:

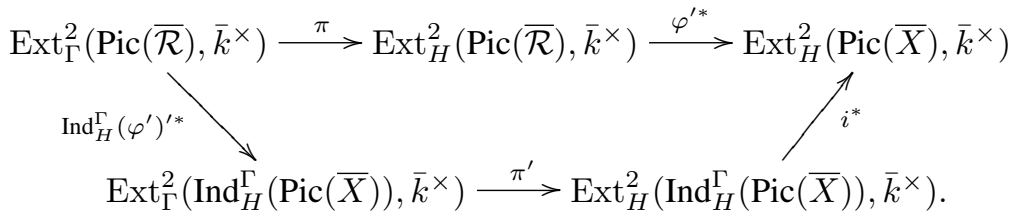

We will prove $\Phi^{*}=\varphi^{*} \circ \pi$ is an isomorphism by proving that $i^{*} \circ \pi^{\prime} \circ \operatorname{Ind}_{H}^{\Gamma}\left(\varphi^{\prime}\right)^{*}$ is an isomorphism and that the diagram above commutes. The latter follows directly from elementary homological reasons.

To prove the former, first observe that $i^{*} \circ \pi^{\prime}$ is an isomorphism by Shapiro's Lemma as it has an inverse $\operatorname{Ind}_{H}^{\Gamma}(\mathrm{id})_{*}^{\prime} \circ \operatorname{Ind}_{H}^{\Gamma}$ with

$$
\begin{aligned}
\operatorname{Ind}_{H}^{\Gamma}: \operatorname{Ext}_{H}^{2}\left(\operatorname{Pic}(\bar{X}), \bar{k}^{\times}\right) \rightarrow \operatorname{Ext}_{\Gamma}^{2}\left(\operatorname{Ind}_{H}^{\Gamma}(\operatorname{Pic}(\bar{X})), \operatorname{Ind}_{H}^{\Gamma}\left(\bar{k}^{\times}\right)\right): & \\
& {[E] \mapsto\left[\operatorname{Ind}_{H}^{\Gamma}(E)\right] }
\end{aligned}
$$

and $\operatorname{Ind}_{H}^{\Gamma}(\mathrm{id})_{*}^{\prime}$ the pushforward

$$
\operatorname{Ext}_{\Gamma}^{2}\left(\operatorname{Ind}_{H}^{\Gamma}(\operatorname{Pic}(\bar{X})), \operatorname{Ind}_{H}^{\Gamma}\left(\bar{k}^{\times}\right)\right) \rightarrow \operatorname{Ext}_{\Gamma}^{2}\left(\operatorname{Ind}_{H}^{\Gamma}(\operatorname{Pic}(\bar{X})), \bar{k}^{\times}\right)
$$

by $\operatorname{Ind}_{H}^{\Gamma}(\mathrm{id})^{\prime}: \operatorname{Ind}_{H}^{\Gamma}\left(\bar{k}^{\times}\right) \rightarrow \bar{k}^{\times}$. This is indeed an inverse by elementary homological reasons.

So it remains to prove $\operatorname{Ind}_{H}^{\Gamma}\left(\varphi^{\prime}\right)^{\prime *}$ is an isomorphism. Therefore we first choose a set of representatives $\left\{\sigma_{1}, \ldots, \sigma_{n}\right\}$ of the classes of $H \backslash \Gamma$ with $\sigma_{1}=$ id.

If Condition (ii) or (ii) is true, then pullback along all components

$$
\psi: \bigoplus_{i=1}^{n} \operatorname{Pic}\left(\sigma_{i} X\right) \rightarrow \operatorname{Pic}(\overline{\mathcal{R}})
$$

is an isomorphism of $H$-modules by Proposition 7 and 8 . We will prove there is a 1-1 correspondence $\tau: \operatorname{Ind}_{H}^{\Gamma}(\operatorname{Pic}(\bar{X})) \rightarrow \bigoplus_{i=1}^{n} \operatorname{Pic}\left(\sigma_{i} X\right)$ and that $\psi \circ \tau=\operatorname{Ind}_{H}^{\Gamma}\left(\varphi^{\prime}\right)^{\prime}$, hence inducing $\operatorname{Ind}_{H}^{\Gamma}\left(\varphi^{\prime}\right)^{\prime}$ is an isomorphism.

First remark that for all $i=1, \ldots, n$, base extension by $\sigma_{i}$ induces a bijection $B_{i}: \operatorname{Pic}(\bar{X}) \rightarrow \operatorname{Pic}\left(\sigma_{i} X\right)$ which does not need to be a $H$-morphism as $H$ does not necessarily commute with $\sigma_{i}$. There are also $H$-morphisms $\psi_{i}: \operatorname{Pic}\left(\sigma_{i} X\right) \rightarrow \operatorname{Pic}(\overline{\mathcal{R}})$ induced by projection on the $i$-th factor, so $\psi=\sum_{i=1}^{n} \psi_{i}$ and $\psi_{1}=\varphi^{\prime}$. It is easy to see the $B_{i}$ and $\psi_{i}$ relate in the following way ${ }^{\sigma_{i}^{-1}} \psi_{i}\left(B_{i}(\mathcal{L})\right)=\psi_{1}(\mathcal{L})$ for any $\mathcal{L} \in \operatorname{Pic}(\bar{X})$.

To define $\tau$, it satisfies defining $\tau(\gamma \otimes \mathcal{L})$ for any $\mathcal{L} \in \operatorname{Pic}(X)$ and $\gamma \in \Gamma$. Suppose $\gamma=\sigma_{i} h$ for $h \in H$ and $1 \leq i \leq n$, then we set $\tau(\gamma \otimes \mathcal{L})$ with 0 as $\left[\sigma_{j}\right]$-components for $j \neq i$ and $B_{i}\left({ }^{h} \mathcal{L}\right)$ as $\left[\sigma_{i}\right]$-component. This is well defined and as all the $B_{i}$ are bijections, $\tau$ is indeed a 1-1 correspondence. Even more

$$
\psi \circ \tau(\gamma \otimes \mathcal{L})=\psi_{i}\left(B_{i}\left({ }^{h} \mathcal{L}\right)\right)={ }^{\sigma_{i}} \psi_{1}\left({ }^{h} \mathcal{L}\right)={ }^{\gamma} \psi_{1}(\mathcal{L})=\operatorname{Ind}_{H}^{\Gamma}\left(\varphi^{\prime}\right)^{\prime}(\gamma \otimes \mathcal{L}) .
$$

So if one of the two conditions holds, $e(X)=0$ if and only if $e(\mathcal{R})=0$. 


\section{Acknowledgement}

The author is supported by Fonds voor Wetenschappelijk Onderzoek Vlaanderen (G.0318.06).

\section{References}

[1] Borovoi, M., Colliot-Thélène, J.L., Skorobogatov, A.: The elementary obstruction and homogeneous spaces. Duke Math. J. 141(2), 321-364 (2008)

[2] Bosch, S., Lütkebohmert, W., Raynaud, M.: Néron Models, Ergebnisse der Mathematik und ihrer Grenzgebiete 3. Folge, vol. 21. Springer Verlag, Berlin (1990)

[3] Colliot-Thélène, J.L., Sansuc, J.: La descente sur les variétés rationnelles. II. Duke Math. J. 54, 375-492 (1987)

[4] Frey, G.: On the structure of the class group of a function field. Arch. Math 38, 33-40 (1979)

[5] Hartshorne, R.: Algebraic Geometry, Graduate Texts in Mathematics, vol. 52. Springer Science+Business Media, Inc., New York (1977)

[6] Mac Lane, S.: Homology, Die Grundlehren der Mathematischen Wissenschaften, vol. 114. Springer Verlag, Berlin (1967)

[7] Milne, J.: Algebraic Geometry, 5th edn. Taiaroa Publishing, Erehwon (2005). http://www.jmilne.org//

[8] Rosenlicht, M.: Toroidal algebraic groups. Proc. Amer. Math. Soc 12, 984-988 (1961)

[9] Wittenberg, O.: On albanese torsors and the elementary obstruction to the existence of 0-cycles of degree 1. Math. Ann. 340(4), 805-838 (2008) 